\title{
Assessment of the inter-rater reliability of the microscopic diagnosis of malaria in three Health Centres of Kayonza District, Eastern Province, Rwanda
}

\author{
Augustin Nzitakera ${ }^{1 *}$, Léonidas Ngizwenayo ${ }^{1}$, Gilbert Niyonshuti ${ }^{1}$, Francine K. Umubyeyi ${ }^{1}$, \\ Christine Mwubahamana ${ }^{1}$, Kato J. Njunwa ${ }^{2}$ \\ ${ }^{1}$ Department of Biomedical Laboratory Science, School of Health Sciences, College of Medicine and Health Sciences, \\ University of Rwanda, Kigali \\ ${ }^{2}$ Director of Research and Post graduate studies, College of Medicine and Health Sciences, University of Rwanda, Kigali
}

\begin{abstract}
Background: Globally, accurate diagnosis of malaria is essential for effective management of malaria and other frequently fatal non-malarial febrile illnesses that share the same signs and symptoms with malaria. Although malaria microscopic diagnosis using stained blood slides is considered to be a standard technique, it suffers subjectivity in its accuracy depending on the knowledge and skill of the technologist reading the slides. The purpose of this retrospective cross-sectional study done in 2013 was therefore to assess the reliability of microscopic diagnosis of malaria using stained blood slides by laboratory technologists in three Kayonza District health centres, Eastern Province, Rwanda. Methods: Forty archived stained blood slides of which 20 had been reported to be positive and 20 negative were selected from each of the three identified health centres by systematic sampling. Overall, 120 stained blood slides were collected and read by the Principal Investigator, and the quality control was done by the National Reference Laboratory. Results and conclusion The results of the diagnosis done by the health centre technologists and principal investigator agreed by $96.67 \%$ and was categorized as almost perfect according to the calculated Cohen's kappa of $0.933, p<0.001$. Still, more trainings are recommended for the technologists.
\end{abstract}

Key words: Microscopic Evaluation, Quality Control, Laboratory Technologist Malaria, Rwanda

\section{Introduction}

Malaria is one of the leading causes of morbidity and mortality in many low income countries particularly in sub-Saharan Africa (Alonso et al., 2011; WHO, 2014). In 2013, out of the 584,000 deaths caused by malaria globally, 90\% occurred in sub-Saharan Africa with 78\% of all deaths affecting children aged less than 5 years (WHO, 2014). Most of the deaths were due to Plasmodium falciparum, although Plasmodium vivax is increasingly recognized as a cause of severe malaria and death (World Health Organization, 2013). With the intensification of malaria control efforts in the last decade, however, malaria has gradually declined as indicated by reduced malaria prevalence, morbidity and mortality around the world, and especially in sub-Saharan African countries (WHO, 2014). Despite the observed decline, malaria transmission continues globally in 43 countries in the African Region of which eight countries, including Rwanda, are on track to achieve reductions in reported malaria case incidences or malaria admission rates of $75 \%$ or more (World Health Organization, 2013).

Treatment of malaria is currently principally based on confirmed parasitological infection and not on the

*Corresponding author: naugustin@khi.ac.rw clinical signs and symptoms, except in situations where parasitological diagnosis is not accessible (WHO, 2010b).

Treatment based on diagnostic testing has several advantages compared to presumptive treatment of all fever episodes. Some of these advantages include: improved care of parasite-positive patients, identification of parasite-negative patients for whom another diagnosis must be sought and be treated accordingly, avoidance of use of antimalarial drugs in parasite-negative patients thereby reducing side effects of drug interactions, and selection pressure for drug resistance, which potentially can result in financial savings on antimalarial drugs, improved public trust in the efficacy of artemisininbased combination therapy (ACT) when it is used only to treat confirmed malaria cases, and improved public trust in the accurate diagnosis and treatment of non-malaria causes of febrile illness (Allen, Hatfield, \& Manyama, 2013; Edson, Glick, \& Massey, 2010; Rafael et al., 2006; WHO, 2011). Microscopic diagnosis of malaria using stained blood slides is considered to be the standard technique. However, the technique relies on the knowledge and skill of the technologist reading the slides and this introduces subjectivity in its accuracy (Amexo, Tolhurst, Barnish, \& Bates, 2004). Studies from various 
countries have reported microscopic misdiagnosis of malaria ranging from $7.8 \%$ over a ten year period in the United States (Edson et al., 2010) to $70.2 \%$ at a local hospital in Tanzania (Allen et al., 2013)

Surprisingly, the subject of quality control and quality assurance of microscopic diagnosis of malaria has for many years not been given adequate consideration for improvement, and this may have inadvertently contributed to the increased number of reported global burden of malarial illnesses (WHO, 2011). Currently however, with a renewed emphasis on malaria elimination and eradication, malaria diagnosis has become a focus of study in order to reduce morbidity and new threat of drug resistance to the currently used antimalarial drugs (WHO, 2011; Wongsrichanalai, Barcus, Muth, Sutamihardja, \& Wernsdorfer, 2007).

With declining malaria prevalence, detection of low grade malaria infections is a challenge due to the low sensitivity of microscopic diagnosis (Alonso et al., 2011; The malERA Consultative Group on Diagnoses and Diagnostics, 2011). The need for accurate detection of the parasite will also be required for monitoring, evaluation and surveillance activities during pre-elimination phase to prevent resurgence of the disease (Alonso et al., 2011). Rwanda, has responded by working to establish and maintaining quality laboratory services for accurate, reliable and timely results as required by set standards (Bates, Bekoe, \& Asamoa-Adu, 2004a; MoH, 2013a, 2014). However, considering that proper maintenance of medical laboratories can be expensive requiring skilled staff, the goal of attaining desired quality control and quality assurance across Rwanda is not yet fully achieved (MoH, 2014).

To improve the accuracy of malaria diagnosis with these existing constraints, Rwanda, through the National Reference Laboratory (NRL), instituted a proficiency testing and evaluation program, regular training of laboratory staff and periodic comparison of malaria microscopy with the Rapid Diagnostic Tests $(\mathrm{MoH}$, 2014). In this program, samples were sent to the national reference laboratory from all District hospitals and from some peripheral health centre laboratories at specified time intervals for confirmation purposes. However, owing to limited resources, the procedure has not reached all the laboratories across the country, and there is decentralization for quality control and quality assurance to be performed at the district level $\mathrm{MoH}$, 2014; WHO, 2009a)

The most commonly used method in detecting errors in diagnosis is to assess the extent to which the scores of observers (i.e. raters) agree with each other, which is called inter-rater agreement. An additional measure called inter-rater reliability indicates the level to which the independent raters can differentiate items under similar conditions (Kottner et al., 2011). Inter-rater reliability measurements use such coefficients as Cohen's kappa because they take into account the random (i.e. chance) agreement that might arise from guessing the scores (Kottner et al., 2011).

The purpose of this retrospective cross-sectional study was therefore to assess the reliability of microscopic diagnosis of malaria using stained blood slides by laboratory technologists in three Kayonza District health centres, Eastern Province, Rwanda in order to identify areas for improvement.

\section{Methods}

A retrospective cross-sectional study using archived stained blood slides was conducted in three health centres in Kayonza district, Eastern Province, Rwanda in September 2012. The Eastern Province is one of the upper moderate transmission areas for malaria in the country $(\mathrm{MoH}, 2013 \mathrm{~b})$. The health centres were selected owing to the large number of malaria patients referred from them to different hospitals in the Province. A minimum sample of 101 stained blood slides, which was raised to 120 to take into account possibly defective smears, was determined from a table of sample size estimation for kappa coefficient detection at 0.05 level of significance, $80 \%$ power, 0.5 proportion of positive diagnosis, and a 2-tailed null hypothesis value of kappa at 0.7 (Sim \& Wright, 2005), based on the time that was available to read all the slides.

Before choosing them into the sample, all records of positive and negative stained blood slides collected between September to November 2012 in each health centre were assigned numbers to be used as sampling frames for systematic sampling (Vos, Strydom, Fouche, \& Delport, 2011). About 40 stained blood slides of which 20 had been reported to be positive and 20 negative were selected from each of the three health centres by systematic sampling to give a total sample of 120 stained blood slides.

The principal investigator who is a trained laboratory technologist from the Biomedical Laboratory Sciences department of the former Kigali Health Institute (KHI), (now part of College of Medicine and Health Sciences, University of Rwanda) carefully read all the blood slides under the CX21 Olympus Microscope. The examination 
of the blood slides was carried out using a high power field magnification (x100 with immersion oil). A result was called positive when malaria parasites were present in the preparation and negative when malaria parasites were absent (WHO, 2010a). The scores were recorded using

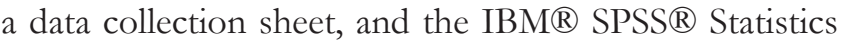
version 20 was utilized for data entry and analysis for percentage agreement between the first and second raters as well as for the Cohen's kappa for inter-rater reliability (McHugh, 2012). To confirm the quality of the results obtained by the principal investigator, $20 \%$ (i.e. 12) of the malaria positive slides and $10 \%$ (i.e. 6 ) of the malaria negative slides were sent to the NRL for confirmation. It is the NRL policy that for quality control, $20 \%$ of positive and $2 \%$ of negative blood slides be sent to the lab for cross-checking; but a little more (i.e. 10\%) of the negative slides were sent. The blood slides included those which were discordant between the health centres and the principal investigator. The NRL and principal investigators results showed a 100\% agreement, and
Cohen's kappa of 1 .

This study was given ethical clearance by the Research Ethics Committee of the former KHI and permission to collect data was obtained from the directors of health centres concerned. All the archived blood slides did not bear names of the patients and thus no inf ormed consent was sought.

\section{Results}

A total of 120 thick film stained malaria slides were examined to assess the level of agreement of malaria diagnosis between the health centre and the principal investigator's diagnosis as shown in Table 1 . The figures indicate that there was a $96.7 \%$ (CI 91.17 - 98.93) agreement between the results from the health centre and those of the principal investigator. The Cohen's kappa for reliability was found to be 0.933 (CI $0.869-0.998$ ), $p$ $<0.001$ which is categorized as almost perfect (Landis \& Koch, 1977; McHugh, 2012)

\section{Table 1. Level of agreement between the sites diagnosis and PI results}

\begin{tabular}{|c|c|c|c|c|c|}
\hline \multirow[b]{2}{*}{ PI } & \multicolumn{3}{|c|}{ Results from the sites } & \multirow[b]{2}{*}{ Cohen's kappa } & \multirow[b]{2}{*}{$p$-value } \\
\hline & Positive & Negative & Total & & \\
\hline Positive & 58 & 2 & 60 & \multirow{3}{*}{$\begin{array}{c}\mathbf{0 . 9 3 3} \\
(\mathrm{CI}: 0.869-0.998)\end{array}$} & \multirow{3}{*}{0.001} \\
\hline Negative & 2 & 58 & 60 & & \\
\hline Total & 60 & 60 & 120 & & \\
\hline
\end{tabular}

\section{Discussion}

In Rwanda, as in many other African countries, the standard diagnostic technique for malaria is the microscopic examination of stained blood slides (WHO, 2010b). As malaria prevalence decreases in many African countries, including Rwanda, the ability to identify all the parasites infections becomes increasingly important. Good quality microscopy conducted by skilled technicians for correct identification of parasites and the ability to manage appropriate quality control, are among the key requirements in the management of malaria (WHO, 2009a). The results of this study indicate that there was an almost perfect agreement in the readings made at the health centres and the principal investigator as reflected by the Cohen's kappa of 0.933 ( $\mathrm{p}<0.001$ ) (McHugh, 2012). The observed high level of agreement could partly be a reflection of the efforts made so far in equipping the laboratories and capacity strengthening for the technologists involved in malaria microscopic diagnosis in Rwanda. For example in 2010/2011 when there was reported a $4.25 \%$ discordance between the blood slide results from the health facilities and the NRL, it was followed by a close corrective supervision of the health facilities by the NRL. The external quality assurance and quality control proficiency testing done between 2010 and 2011 obtained acceptable scores between $80 \%$ and $97.5 \%$, well above the minimum acceptable score of $75 \%$ (MoH, 2013c).

Despite an almost perfect agreement between the readers detected in this study, the two positive slides scored as negative by the health centres is worth further consideration in the context of decreasing malaria prevalence in Rwanda. A few studies have shown that the malaria prevalence, estimated as the proportion of stained blood slides found to have malaria parasites, tend to vary considerably at low parasite densities ranging between $4-100 / \mu$ l of blood, but much less so above that density (Billo et al., 2013; Harris et al., 2010; McKenzie, Sirichaisinthop, Miller, Gasser, \& Wongsrichanalai, 2003). There are some known factors that adversely affect the slide reading results at low parasite density levels. These include quality of slide preparation, quality of reagents and microscopes, random selection of microscope fields, level of training, and motivation and concentration including workload of the microscopists as well as to whether context is clinical or research (Billo et al., 2013; 
Harris et al., 2010; McKenzie et al., 2003). For example, the malaria microscopist technologists located in the malaria endemic areas are faced with large numbers of blood slides to read daily (Tangpukdee, et al., 2009), which tend to decrease their ability to perform accurately and with precision (World Health Organzation, 2009). The preparation of thick blood films has been reported to lose up to $60 \%$ of the parasites, which is a significant barrier to accurate microscopic diagnosis of malaria (Dowling \& Shute, 1966). Nevertheless, with a 6 months of training technologists have been shown to increase the malaria microscopy evaluation accuracy with a notable improvement in scores from $84 \%$ to $91 \%$, (Bates, Bekoe, \& Asamoa-Adu, 2004b).

Although comparative studies show that other techniques such as the polymerase chain reaction (PCR) can detect parasites missed by microscopic diagnosis for densities below 40-50 parasites/ $\mu$ l, (Kilian et al., 2000), they have a limitation of being too expensive and technically demanding to be used in a routine diagnostic laboratory. Evidently, the efforts to perfect the microscopic diagnosis of malaria at the district and health centre levels remain crucial and may continue to be like that for quite some time in the future.

\section{Limitation of the study}

The limitation of the study relates to the fact that Cohen kappa does not have clear-cut interpretation with respect to reliability, although it is generally stated that a value of 0.7 and above is acceptable and below it is weak. Where the goal is toward elimination of malaria, a value of kappa $=1.0$ remains the target.

\section{Conclusion}

The results of this study have shown that the degree of reliability of results from the three health centres in Kayonza is almost perfect. It is asserted that such achievement might be attributed to the deliberate plans and efforts made to improve microscopic diagnosis in Rwanda through regular trainings of technologists, supply of equipment and reagents within the means available and engaging in corrective supervision of certain health facilities having low reliability scores. (MoH, 2013c; WHO, 2009b, 2011). The quality of care with respect to malaria management in the country, ultimately rests with accurate diagnosis of malaria at all levels. Given that microscopy is still the mainstay of the diagnosis, more resources will need to be available to improve further and sustain the current level of accuracy in order to save more lives and thus build more trust between the health care system and the patients.

\section{Authors' contributions}

NA was responsible for designing and implementing the study, analysing the data and Writing the manuscript. NL, NG, UK F and MC were involved in data collection process. KJN was the mentor and supervisor of this research.

\section{Acknowledgements}

This research was made possible with technical assistance of the co-authors. "This work was supported in part by Africa Research Initiatives and Support Enterprise (ARISE), grant number W.07.10.102 funded by Netherlands African Partnership for Clinical Trials and Capacity Building Program (NACCAP) of The Netherlands Organisation for Scientific Research The Netherlands Foundation for the Advancement of Tropical Research (NWO/WOTRO). Its contents are solely the responsibility of the authors and do not necessarily represent the official views of the supporting offices."

\section{Declaration}

The authors declare no conflict of interest in relation to presentation of this manuscript.

\section{References}

Allen, L. K., Hatfield, J. M., \& Manyama, M. J. (2013). Reducing microscopy-based malaria misdiagnosis in a low-resource area of Tanzania. Tanzania Journal of Health Research, 15(1), 1-9. doi:10.4314/thrb.v15i1.4

Alonso, P. L., Brown, G., Arevalo-Herrera, M., Binka, F., Chitnis, C., Collins, F., Tanner, M. (2011). A research agenda to underpin malaria eradication. PLoS Medicine, 8(1), e1000406. doi:10.1371/journal.pmed.1000406

Amexo, M., Tolhurst, R., Barnish, G., \& Bates, I. (2004). Malaria misdiagnosis: Effects on the poor and vulnerable. Lancet, 364(9448), 1896-1898. doi:10.1016/S0140-6736(04)17446-1

Bates, I., Bekoe, V., \& Asamoa-Adu, A. (2004a). Improving the accuracy of malaria-related laboratory tests in Ghana. Malaria Journal, 3, 38. doi:10.1186/1475-2875-3-38

Bates, I., Bekoe, V., \& Asamoa-Adu, A. (2004b). Improving the accuracy of malaria-related laboratory tests in Ghana. Malaria Journal, 3, 38. doi:10.1186/1475-2875-3-38

Billo, M. A., Diakité, M., Dolo, A., Diallo, M., Poudiougou, B., Diawara, S. I., Doumbo, O. K. (2013). Inter-observer agreement according to malaria parasite density. Malaria Journal, 12, 335. doi:10.1186/1475-2875-12-335

Dowling, C. M. A., \& Shute, G. T. (1966). A Comparative Study of Thick and Thin Blood Films in the Diagnosis of Scanty Malaria Parasitaemia. Bulletin of the World Health Organization, 34, 249-267. Retrieved from http:// www.ncbi.nlm.nih.gov/pmc/articles/PMC2475932/pdf/ bullwho00611-0089.pdf 
Edson, D. C., Glick, T., \& Massey, L. D. (2010). Detection and Identification of Malaria Parasites: A Review of Proficiency Test Results and Laboratory Practices. Laboratory Medicine, 41(12), 719-723. doi:10.1309/LM0KC4BEYHGDSZCU

Harris, I., Sharrock, W. W., Bain, L. M., Gray, K.-A., Bobogare, A., Boaz, L., Cheng, Q. (2010). A large proportion of asymptomatic Plasmodium infections with low and submicroscopic parasite densities in the low transmission setting of Temotu Province, Solomon Islands: challenges for malaria diagnostics in an elimination setting. Malaria Journal, 9, 254. doi:10.1186/1475-2875-9-254

Kilian, A. H. D., Metzger, W. G., Mutschelknauss, E. J., Kabagambe, G., Langi, P., Korte, R., \& Sonnenburg, F. (2000). Reliability of malaria microscopy in epidemiological studies: results of quality control. Tropical Medicine and International Health, 5(1), 3-8. doi:10.1046/j.1365-3156.2000.00509.x

Kottner, J., Audige, L., Brorson, S., Donner, A., Gajewski, B. J., Hroóbjartsson, A., Streiner, D. L. (2011). Guidelines for Reporting Reliability and Agreement Studies (GRRAS) were proposed. International Journal of Nursing Studies, 48(6), 661671. doi:10.1016/j.ijnurstu.2011.01.016

Landis, J. R., \& Koch, G. G. (1977). The measurement of observer agreement for categorical data. Biometrics, 33 , 159-174.

McHugh, M. L. (2012). Interrater reliability: the kappa statistic. Biochemia Medica, 22(3), 276-282. Retrieved from http:/ / hrcak.srce.hr/index.php?show=clanak\&id_clanak_ jezik $=132393$

McKenzie, F. E., Sirichaisinthop, J., Miller, R. S., Gasser, R., \& Wongsrichanalai, C. (2003). Dependence of Malaria Detection and Species Diagnosis by Microscopy on Parasite Density, 69(4), 372-376. Retrieved from http://hinarilogin. research4life.org/uniquesigwww.ajtmh.org/uniquesig0/ content/69/4/372.full.pdf + html

MoH. (2013a). Annual Report on the Implementation of The East Africa Public Health Laboratory Networking ( EAPHLN ) Project. Kigali-Rwanda: MoH. Retrieved from http://www.eaphln-ecsahc.org/rwanda/index.php/ publications / reports?download=484:report-eaphlnp-july2012-june-2013-final

MoH. (2013b). National Strategic Plan for Integrated Vector Management (IVM) (2013-2017). KIgali: MoH.

MoH. (2013c). PRESIDENT'S MALARIA INITIATIVE Malaria Operational Plan (MOP) Rwanda FY 2013. KIGALI. Retrieved from http://www.pmi.gov/docs/default-source/ default-document-library/malaria-operational-plans/fy13/ rwanda_mop_fy13.pdf?sfvrsn=8BOOK FORMAT/ REFERENCES/ALL REFERENCES/rwanda_mop_fy 2013.pdf

MoH. (2014). PRESIDENT 'S MALARIA INITLATIVE Rwanda Malaria Operational Plan FY 2014. KIGALI. Retrieved from http://www.pmi.gov/docs/default-source/ default-document-library/malaria-operational-plans/fy14/ rwanda_mop_fy14.pdf?sfvrsn $=8$
Rafael, M. E., Taylor, T., Magill, A., Lim, Y.-W., Girosi, F., \& Allan, R. (2006). Reducing the burden of childhood malaria in Africa: the role of improved. Nature, 444 Suppl , 39-48. doi: $10.1038 /$ nature 05445

Sim, J., \& Wright, C. C. (2005). The kappa statistic in reliability studies: use, interpretation, and sample size requirements. Physical Therapy, 85(3), 257-68. doi:15733050

Tangpukdee, N., Duangdee, C., Wilairatana, P., \& Krudsood, S. (2009). Malaria Diagnosis: A Brief Review. The Korean Journal of Parasitology, 47(2), 93. doi:10.3347/kjp.2009.47.2.93

The malERA Consultative Group on Diagnoses and Diagnostics. (2011). A research agenda for malaria eradication: diagnoses and diagnostics. PLoS Medicine, 8(1), e1000396. doi:10.1371/journal.pmed.1000396

Vos, A. de, Strydom, H., Fouche, C., \& Delport, C. (2011). Research at grass roots for the social sciences and buman service professions (4th ed.). Pretoria: Van Schaik.

WHO. (2009a). Malaria Microscopy Quality assurance Mannual Version 1. Geneva: WHO. Retrieved from http://www. who.int/malaria/publications/malaria_microscopy_QA_ manual.pdf

WHO. (2009b). Parasitological confirmation of malaria diagnosis: WHO technical consultation, Geneva, 6-8 October 2009. Retrieved from http://whqlibdoc.who.int/ publications/2010/9789241599412_eng.pdf

WHO. (2010a). Basic Malaria Microscopy Part I. Learner's guide (2nd ed.). Geneva: WHO. Retrieved from http://whqlibdoc. who.int/publications/2010/9789241547826_eng.pdf

WHO. (2010b). Guidelines for the Treatment of Malaria Second edition. Geneva: WHO. Retrieved from http://whqlibdoc. who.int/publications/2010/9789241547925_eng.pdf

WHO. (2011). Universal access to malaria diagnostic testing An Operational Manual. Geneva: World Health Organization. Retrieved from http://www.paho.org/hq/index. php?gid=20083\&option $=$ com_docman\&task=doc_view

WHO. (2014, February). World Malaria Report 2014. Geneva: WHO. doi:10.1007/s00108-013-3390-9

Wongsrichanalai, C., Barcus, M. J., Muth, S., Sutamihardja, A., \& Wernsdorfer, W. H. (2007). A Review of Malaria Diagnostic Tools : Microscopy and Rapid Diagnostic Test ( RDT ). Am. J. Trop. Med. Hyg, 77(Suppl 6), 119-127. Retrieved from http://www.ncbi.nlm.nih.gov/books/NBK1695/pdf/ pg119.pdf

World Health Organization. (2013). World Malaria Report. Geneva. 